\title{
BLICKDIAGNOSE
}

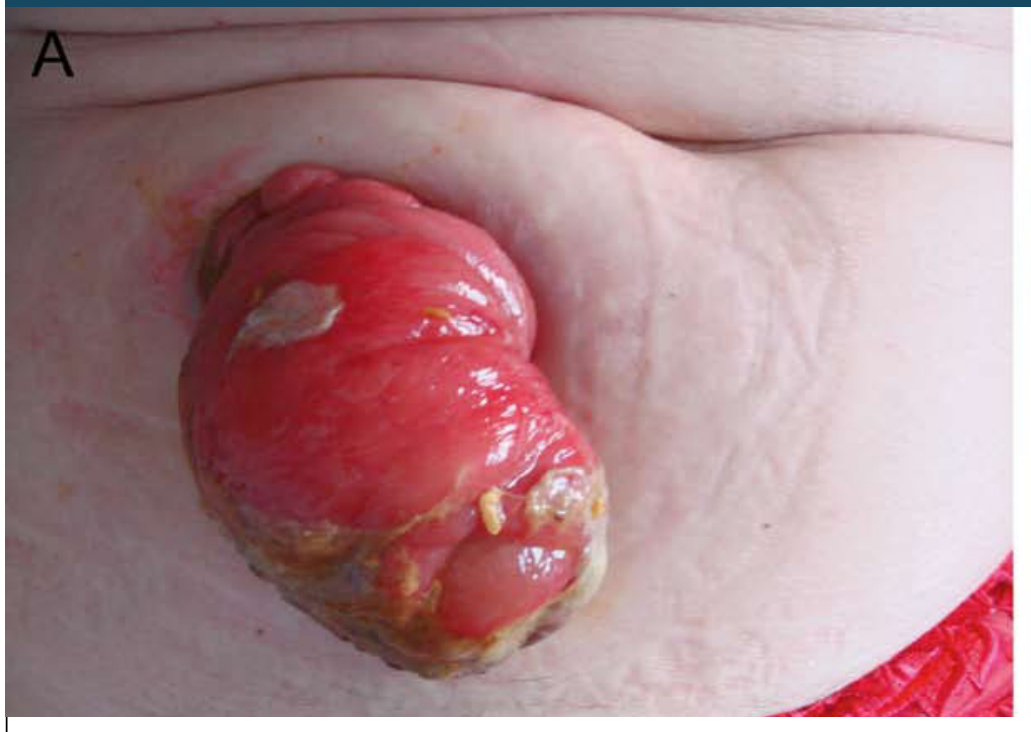

Fortgeschrittenes Sigmakarzinom

\section{Wie versorgt man dieses Kolostoma?}

Die Patientin stellte sich in reduziertem Allgemein- und kachektischem Ernährungszustand in der Chirurgischen Poliklinik vor. Anamnestisch sei es in den vergangenen Tagen zu einer zunehmenden Vergrößerung des Kolostomas gekommen, was der Patientin nun Schwierigkeiten bei der Versorgung bereite.

— Vor einigen Wochen war ein diffus metastasiertes Sigmakarzinom diagnostiziert worden. Die Patientin hatte jede systemisch-onkologische Therapie abgelehnt. Es wurde lediglich bei lokaler Tumorstenose ein palliatives doppelläufiges Transversostoma angelegt.

Die Abbildung zeigt einen Stomaprolaps, wie er bei 1-3\% der Patienten mit Ileo- oder Kolostomie auftreten kann. Im vorliegenden Fall war es durch zunehmenden Aszites bei Peritonealkarzinose zu einem Vorfall der abführenden Schlinge gekommen.

Die operative Revision mit Resektion des prolabierten Darmanteils und/oder die Einengung des Bauchdeckendurchtritts sind hier die definitive Therapie der Wahl. In palliativen Situationen kann eine manuelle Kompression und anschließende Reposition der ödematös geschwollenen Darmschlinge erfolgen und durch eine Leibbinde mit sog. Stomakappe vorübergehend gesichert werden.

Nicht verwechselt werden sollte ein Stomaprolaps mit dem gewollten evertierten und prominenten $(3-4 \mathrm{~cm}$ ) Ileostoma (bei einem Kolostoma sind 0,5-1,0 cm ausreichend). Die erst 1952 durch Brooke eingeführte Technik erlaubt eine direkte Ableitung des aggressiven Dünndarmstuhls in den Stomabeutel und verhindert so therapierefraktäre peristomale Hautmazerationen.
Abb. A Ödematös geschwollener Transversostomaprolaps vor manueller Kompression und Reposition. Abb. B Durch Auflage der evertierten Darmschleimhaut auf dem Stomabeutel ist es bereits punktuell und flächig zu Ulzerationen der Schleimhaut gekommen $(*)$. Die zuführende (= stuhlführende) Öffnung des doppelläufigen Stomas ist durch den Prolaps des abführenden Darmabschnittes nahezu vollständig verlegt (Pfeil).

Keywords: prolabs of the colostomy -Priv.-Doz. Dr. med. Philipe N. Khalil, Cand. med. Jana Eisenlohr, Dr. med. Axel Kleespies, Chirurgische Klinik und Poliklinik - Innenstadt, Klinikum der LMU, Nußbaumstraße 20, D-80336 München

\section{Ihr besonderer Fall?}

\section{Stellen Sie uns Ihren Fall vor}

Sicher sehen auch Sie ab und an einen besonders eindrucksvollen Befund in Ihrer Praxis. Fotografieren Sie ihn, schreiben Sie uns unter dem Stichwort Blickdiagnose, bei Veröffentlichung erhalten Sie 100 Euro.

MMW-Fortschritte der Medizin E-Mail:manhart@urban-vogel.de Fax: 089/203043-31424 